\title{
(2) OPEN ACCESS \\ Identifying best modelling practices for tobacco control policy simulations: a systematic review and a novel quality assessment framework
}

\author{
Vincy Huang (1) , Anna Head, Lirije Hyseni, Martin O'Flaherty, lain Buchan, \\ Simon Capewell, Chris Kypridemos
}

\begin{abstract}
- Additional supplemental material is published online only. To view, please visit the journal online (http://dx.doi. org/10.1136/tobaccocontrol2021-056825).
\end{abstract}

Department of Public Health, Policy and Systems, University of Liverpool, Liverpool, UK

\section{Correspondence to} Vincy Huang, Department of Public Health, Policy and Systems, University of Liverpool, Liverpool L69 3GB, UK; vincyhwj@liverpool.ac.uk

Received 1 June 2021 Accepted 27 December 2021
Check for updates

(C) Author(s) (or their employer(s)) 2022. Re-use permitted under CC BY. Published by BMJ.

To cite: Huang $V$, Head $A$, Hyseni L, et al. Tob Control Epub ahead of print: [please include Day Month Year]. doi:10.1136/

tobaccocontrol-2021-056825

\section{ABSTRACT}

Background Policy simulation models (PSMs) have been used extensively to shape health policies before real-world implementation and evaluate postimplementation impact. This systematic review aimed to examine best practices, identify common pitfalls in tobacco control PSMs and propose a modelling quality assessment framework.

Methods We searched five databases to identify eligible publications from July 2013 to August 2019. We additionally included papers from Feirman et al for studies before July 2013. Tobacco control PSMs that project tobacco use and tobacco-related outcomes from smoking policies were included. We extracted model inputs, structure and outputs data for models used in two or more included papers. Using our proposed quality assessment framework, we scored these models on population representativeness, policy effectiveness evidence, simulated smoking histories, included smoking-related diseases, exposure-outcome lag time, transparency, sensitivity analysis, validation and equity. Findings We found 146 eligible papers and 25 distinct models. Most models used population data from public or administrative registries, and all performed sensitivity analysis. However, smoking behaviour was commonly modelled into crude categories of smoking status. Eight models only presented overall changes in mortality rather than explicitly considering smoking-related diseases. Only four models reported impacts on health inequalities, and none offered the source code. Overall, the higher scored models achieved higher citation rates.

Conclusions While fragments of good practices were widespread across the reviewed PSMs, only a few included a 'critical mass' of the good practices specified in our quality assessment framework. This framework might, therefore, potentially serve as a benchmark and support sharing of good modelling practices.

\section{INTRODUCTION}

Since 2020, it became evident that COVID-19 modelling had influenced, and on occasions dictated, disease control policies to shape the subsequent course of the pandemic. ${ }^{1}$ For decades before this publicity, policy simulation models (PSMs) have been applied to inform evidence-based health policymaking and had contributed to many successful tobacco control policies. ${ }^{2}$

Various actions have been taken to end the tobacco pandemic, which killed over 100 million people worldwide during the 20th century. ${ }^{3}$ These actions notably include policies targeting the accessibility, acceptability and affordability of tobacco products. Tobacco control models have been used extensively to shape such policies, both prior to real-world implementation and also to evaluate post-implementation impact. ${ }^{4}$

Two recent systematic reviews identified a plethora of tobacco control models intended for policymaking and policy evaluation. ${ }^{5-7}$ This is a very active research area, reflecting an explosion of available data, novel methodologies and low-cost, widely available computational power. ${ }^{8}$ However, this plethora of independently developed models may represent an unnecessary effort in replication compared with a more collaborative approach. Second, neither previous systematic review examined model quality, which perhaps reflects a lack of an appropriate quality assessment framework for simulation models.

Although several publicly available quality assessment tools exist, including Consolidated Health Economic Evaluation Reporting Standards (CHEERS) checklist, ${ }^{9}$ Grading of Recommendations, Assessment, Development and Evaluations (GRADE), ${ }^{10}$ and the National Institute for Health and Care Excellence (NICE) Methodology Guide quality checklist, ${ }^{11}$ none appear well suited for the diversity of tobacco control models. The NICE and CHEERS checklists are designed mainly to evaluate economics models, while the GRADE guideline focuses mainly on evidence certainty and is not topic specific.

The lack of such an applicable framework partly reflects the fast evolution of modelling approaches, the multidisciplinary nature of modelling and the multitude of questions models are asked to address. While developing a generic quality assessment framework for simulation models appears challenging, developing a domain-specific one for tobacco control simulation models might represent a more feasible first step.

We, therefore, defined two aims for this study:

1. To assess the modelling practices used in tobacco control PSMs (reviewing both best practices and common limitations).

2. To produce a quality assessment framework appropriate for tobacco control PSMs to potentially improve future policy modelling practices.

\section{METHODS}

\section{Study design}

We systematically reviewed the published tobacco control PSMs, particularly evaluating their methodological strengths and weaknesses. We then 
critically appraised and compared their modelling practices with an ideal but feasible tobacco control PSM prototype.

We report the results following the Synthesis Without MetaAnalysis statement (online supplemental table S1), ${ }^{12}$ and present our findings in compliance with the Preferred Reporting Items for Systematic Reviews and Meta-Analysis statement. ${ }^{13}$

\section{Definitions}

PSMs: quantitative frameworks that integrate evidence from cross-disciplinary sources to estimate the impact of existing or planned policies.

Tobacco control PSMs: PSMs that estimate existing or planned tobacco control policies impact.

Smoking-related diseases: diseases widely accepted to be causally linked to smoking, including chronic obstructive pulmonary disease (COPD), cardiovascular disease and common cancers.

External validation: comparing the model result with actual observed data not used as model inputs. ${ }^{14} 15$

Cross-validation: comparison of results between models which address the same problem. ${ }^{14} 15$

Sensitivity analysis: studying the model output changes caused by varying model inputs. ${ }^{14} 15$

\section{Search strategy}

We included the studies in the Feirman et al systematic review (to July 2013) ${ }^{6}$ and extended the search strategy to cover the period to September 2019.

We searched five electronic databases (Embase, EconLit, PsycINFO, PubMed and CINAHL Plus). The search keywords for five databases are detailed in online supplemental text S1. We also scanned the reference lists of all included studies for potential additional papers.

\section{Study selection and inclusion criteria}

Inclusion criteria:

1. Referred to any tobacco product or tobacco use.

2. Contained peer-reviewed tobacco control PSMs that projected tobacco use and tobacco-related outcomes from tobacco control policy scenarios.

3. The model was reported in at least two peer-reviewed studies.

4. Full text in English.

We assessed the retrieved studies using the Participants, Interventions, Comparators, Outcomes and Study design approach (online supplemental table S2).

Two reviewers ( $\mathrm{VH}$ and $\mathrm{AH}$ ) independently screened titles and abstracts for eligibility using the inclusion and exclusion criteria, then screened the full text of all potential eligible papers. A third reviewer $(\mathrm{CK})$ with modelling expertise was consulted to resolve any discrepancies. We used Zotero, reference management software, for screening.

We registered the protocol for our study with PROSPERO (CRD42020178146) and published it separately. ${ }^{16}$

\section{Data extraction}

We used a predefined and piloted data extraction form (online supplemental table S3) to extract study information on:

1. General information (ie, model name, code license, conflict of interest (COI)).

2. Model simulation methods.

3. Modelled population sociodemographic characteristics (ie, age, gender, ethnicity/race, socioeconomic status).

4. Risk factors.
5. Included diseases.

6. Data sources used.

7. Model outcome types (ie, health, economics).

8. Model checking, transparency, validation and calibration.

9. Model limitations reported.

We assessed data extraction quality by allowing a second reviewer to double-check $50 \%$ of the extraction forms for accuracy and completeness.

\section{Evidence synthesis}

We grouped the extracted study data by model name when reported or by the first author of the earliest publication.

We critically reviewed model data inputs, epidemiological principles, assumptions, transparency and whether they reported (a) relevant sources of parametric uncertainty, (b) potential limitations, (c) model validations and sensitivity analyses, and (d) technical documentation.

\section{A proof-of-concept quality assessment framework}

We then developed a simple quality assessment framework for model inputs, structure and outputs based on potential Good Modelling Practices (detailed in online supplemental text S2).

One point was given when each of the described criteria below was met:

- Population: model population data are representative of the population that the modelled policies will apply to.

- Policy effectiveness: the policy effectiveness data were extracted from empirical evidence.

- Smoking status: the model captured the cumulative effect of smoking (smoking intensity, smoking history, quitting age, etc).

- Smoking-related diseases: the model estimated the effect on the majority of important smoking-related diseases (quantifying both morbidity and mortality).

- Lag time: the model explicitly captured the time lag between exposure and disease onset.

- Transparency: technical or non-technical documents available to provide model transparency.

- Uncertainty/sensitivity analysis performed and reported.

- Validation: the model was validated.

- Equity: the model explored the equity impact of policies.

\section{RESULTS}

The search initially identified 5046 articles. After removing duplicates and screening titles and abstracts, 441 articles were eligible for full-text review. In total, 146 studies met the inclusion criteria and were included for data extraction, including 9 additional studies identified from included studies' reference lists (figure 1). ${ }^{17-25}$

We identified a total of 25 eligible tobacco control PSMs (table 1 and online supplemental text S3). Five models were used by only one paper in our searches. Nevertheless, we included them in our study because they were also used in papers published before July 2013, as identified in the Feirman et al review. The five models were Chronic Disease Model (CDM), Coronary Heart Disease Policy (CHD Policy) Model, Lung Cancer Policy Model (LCPM), Mendez model and Mejia model.

The SimSmoke model appeared to be the most used model with 18 peer-reviewed studies, ${ }^{24} 26-42$ and the Burden of Disease Epidemiology, Equity and Economics $\left(\mathrm{BODE}^{3}\right)$ model ranked second with 11 peer-reviewed studies. ${ }^{20} 2543-51$

Cohort (macro)simulation and microsimulation approaches were the most used self-reported methodologies. Agent-based 

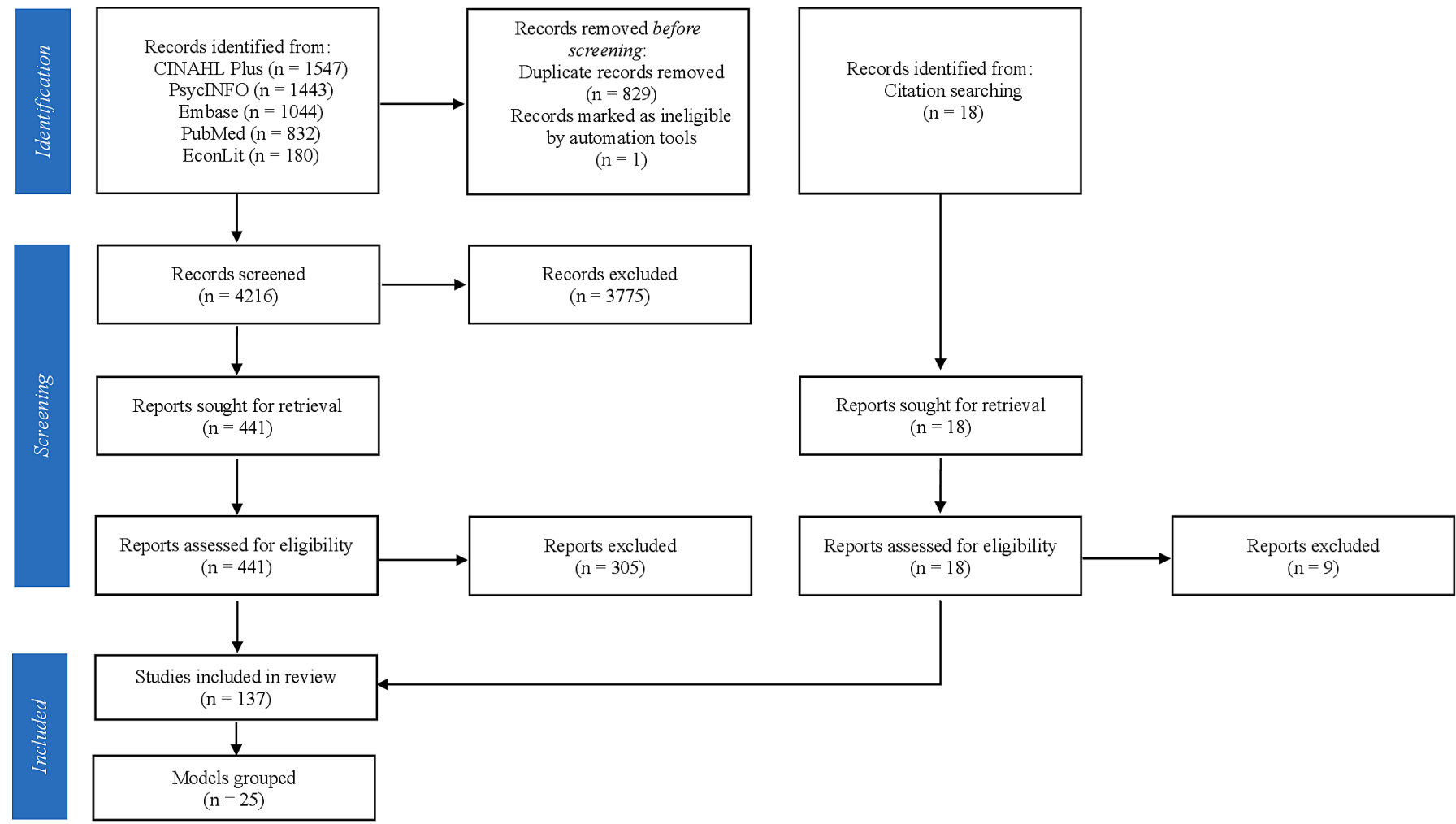

Figure 1 An adapted Preferred Reporting Items for Systematic Reviews and Meta-Analysis flow chart of identified studies.

modelling was used in just one model (Tobacco Town), likewise system dynamics in Prevention Impacts Simulation Model.

The diversity of model outcomes reflected the wide range of model purposes. Nineteen models reported health economics outcomes, 22 reported health measures including mortality or morbidity, with just one (ModelHealth: Tobacco) reporting hospital admissions (figure 2, online supplemental table S4). Only four models reported the policy impact on equity: $\mathrm{BODE}^{3}$, CDM, extended cost-effectiveness analysis (ECEA) tobacco tax model and IMPACT.

Of the eligible 146 studies, 5 tobacco industry-funded studies reported COI or commercial funding. ${ }^{52-56}$ However, we did not further investigate inaccurate or incomplete COI reporting.

\section{Good Modelling Practices}

\section{Model inputs}

Twenty-one of the 25 models appropriately used population data from public or administrative registries. Conversely, three used information from randomised controlled trial (RCT) participants that are rarely population representative. ${ }^{17}$ 57-62

Eleven out of 25 models used systematic reviews or metaanalyses to inform policy effectiveness in the model. Six models used treatment-specific RCT values to estimate policy effectiveness. ${ }^{17}$ 57-69 Five models assumed the policy effectiveness by project teams or expert opinions. ${ }^{18} 2426-4252$ 70-78 In particular, SimSmoke, the most frequently referenced model, used the policy effect size provided by experts, likewise the ECEA tobacco tax model. Furthermore, three models calculated policy effectiveness from government reports or surveys. ${ }^{79-83}$ Similarly, Population Health Impact Model estimated policy effectiveness by simple assumptions.
Model structure

Abiding by fundamental epidemiological principles, a tobacco simulation model structure should ideally aim to: (a) capture the cumulative effect of smoking (ie, for lung cancer and COPD ${ }^{84-86}$ ), including the intensity, duration, initiation and cessation; (b) estimate the effect on the main smoking-related diseases (ideally including both morbidity and mortality $)^{87}$; (c) capture the time lag between exposure and disease risk $^{87}$; (d) be transparent. ${ }^{1488}$

Six models simulated smoking histories (including pack-years, pack-days) or quitting histories. ${ }^{2426-42 ~ 69-74798089-93}$ A further 19 models considered smoking only as a categorical exposure (ie, never/ex/current smoker).

Lag time was reported in 11 out of 25 . $^{17182024-52575875-77818292-104}$ These models either estimated relative risk decline by time since cessation or cost decay by quit years. The remaining models did not report any considerations on the effect of time since cessation.

We summarised the number of diseases included in each model in figure 3 and online supplemental table S5. Models varied in how well they reflected epidemiology pathways (online supplemental table S6). Seventeen used smoking-related diseases to generate smoking-related outcomes. Two models calculated all-cause mortality directly from smoking status. The remaining six models estimated their outcomes directly, using the number of smokers or non-smokers, without explicitly modelling disease pathways.

\section{Transparency}

Nineteen models provided model documentation to explain model technical details for readers (all except Benefits of Smoking Cessation on Outcomes model, Baker model, Cantor 
Table 1 Model summary (in descending order of the number of peer-reviewed articles)

\section{Model name/first author: SimSmoke}

Model type (self-reported): discrete Markov model, macrosimulation

Risk factors included (smoking status provided with details): smoking status (never,

former, current), years since quitting

Diseases: NA

Outcomes: mortality, smoking prevalence, maternal and child health outcomes (smokingattributable low birth weight, preterm births and sudden infant death syndrome) cases, uncertainty

Sensitivity analysis: performed sensitivity analysis

Validation: External validation

Number of peer-reviewed articles in this search: 18

Related papers: $2426-42$

\section{Model name/first author: IMPACT}

Model type (self-reported): cell-based model

Risk factors included (smoking status provided with details): blood pressure, cholesterol, diabetes, fruit and vegetable, smoking status (never smoker, long-term exsmoker, recent ex-smoker, current smoker), salt intake, saturated fat intake, BMI, physical activities

Diseases: CHD, T2DM

Outcomes: equity, CHD mortality, smoking prevalence, life-years gained, uncertainty

Sensitivity analysis: Monte Carlo simulation

Validation: external validation

Number of peer-reviewed articles in this search: 6

Related papers: 23 107-111

Model name/first author: EQUIPTMOD

Model type (self-reported): Markov state-transition cohort model, macrosimulation

Risk factors included (smoking status provided with details): smoking status

(former, current)

Diseases: COPD, CHD, stroke, lung cancer

Outcomes: cost, ROI, ICER, QALY

Sensitivity analysis: univariate, others

Validation: no model validation

Number of peer-reviewed articles in this search: 5

Related papers: $112-116$

Model name/first author: Benefits of Smoking Cessation on Outcomes model

Model type (self-reported): discrete-time Markov model

Risk factors included (smoking status provided with details): smoker, recent quitter and long-term quitter

Diseases: COPD, CHD, stroke, lung cancer, asthma exacerbation, chronic obstructive lung diseases

Outcomes: total morbidity and mortality, economics impact

Sensitivity analysis: one-way, PSA

Validation:no model validation

Number of peer-reviewed articles in this search: 4

Related papers: $63-66$

Model name/first author: Johansson model

Model type (self-reported): a Markov model

Risk factors included (smoking status provided with details): smoking status (former, current)

Diseases: COPD, CHD, stroke, cancers

Outcomes: QALY, life years lost, cost, uncertainty

Sensitivity analysis: univariable, multivariable, PSA

Validation: external validation

Number of peer-reviewed articles in this search: 3

Related papers: 175758
Model name/first author: Burden of Disease Epidemiology, Equity and Economics model

Model type (self-reported): a proportional multistate life-table, macrosimulation, Markov model

Risk factors included (smoking status provided with details): smoking status (never, former, current)

Diseases: 16 diseases-CHD, stroke, COPD, lower respiratory tract infection, and multiple cancers: lung, oesophageal, stomach, liver, head and neck, pancreas, cervical, bladder, kidney, endometrial, melanoma, and thyroid (with smoking protecting against the latter three cancers)

Outcomes: equity, health-systems cost-savings, smoking prevalence, QALYs gained, health-adjusted life years, uncertainty

Sensitivity analysis: PSA

Validation: cross-validation, external validation

Number of peer-reviewed articles in this search: 11

Related papers: 2025 43-51

Model name/first author: extended cost-effectiveness analysis (ECEA) tobacco tax model

Model type (self-reported): ECEA

Risk factors included (smoking status provided with details): smoking

prevalence, number of cigarettes smoked daily; age at quitting

Diseases: COPD, heart disease, stroke, lung cancer, bladder cancer

Outcomes: disease treatment costs, averted premature death, life-years gained

additional revenues generated, equity, uncertainty

Sensitivity analysis: one-way, Monte Carlo simulation

Validation: validated model

Number of peer-reviewed articles in this search: 5

Related papers: $70-74$

Model name/first author: DYNAMO-HIA model

Model type (self-reported): macrosimulation, Markov-based life-table

Risk factors included (smoking status provided with details): alcohol intake,

$\mathrm{BMI}$, smoking status, secondhand smoking

Diseases: COPD, IHD, stroke, cancers, T2DM

Outcomes: mortality, morbidity, morbidity-free years, life expectancy, number of deaths

Sensitivity analysis: performed sensitivity analysis

Validation: no model validation

Number of peer-reviewed articles in this search: 5

Related papers: $94-98$

Model name/first author: Jiménez model

Model type (self-reported): closed cohort Markov model

Risk factors included (smoking status provided with details): smoking status, willingness to quit history

Diseases: COPD, CVD, T2DM

Outcomes: incremental cost-savings, number of quitters

Sensitivity analysis: univariate sensitivity analysis

Validation: internal validation

Number of peer-reviewed articles in this search: 3

Related papers: 89-91

Model name/first author: Prevention Impacts Simulation Model

Model type (self-reported): system dynamics model

Risk factors included (smoking status provided with details): blood pressure, cholesterol, secondhand smoking, obesity, psychological distress, fruit and vegetable, smoking status (never smoker, long-term ex-smoker, recent ex-smoker, current smoker), blood glucose categories (normal, pre-diabetic, diabetic), periodontal disease, sleep apnoea, small particulate air pollution and inadequate use of aspirin for primary prevention

Diseases: CVD

Outcomes: mortality and morbidity, healthcare cost, productivity loss, uncertainty Sensitivity analysis: PSA

Validation: external validation

Number of peer-reviewed articles in this search: 3

Related papers: $75-77$ 
Table 1 Continued

Model name/first author: Baker model

Model type (self-reported): closed cohort Markov model

Risk factors included (smoking status provided with details): eligible smoker, ineligible smoker, initial quitter, successful quitter

Diseases: NA

Outcomes: number of quitters, morbidity, mortality, medical expenditures

Sensitivity analysis: univariate, multivariable analyses

Validation: no model validation

Number of peer-reviewed articles in this search: 2

Related papers: 99100

Model name/first author: Cantor model

Model type (self-reported): decision-analytical model

Risk factors included (smoking status provided with details): smoking status

Diseases: NA

Outcomes: cost, QALY

Sensitivity analysis: one-way, two-way uncertainty analyses

Validation: no model validation

Number of peer-reviewed articles in this search: 2

Related papers: 6162

\section{Model name/first author: Cost-Effectiveness of Preventing AIDS Complications-US} model

Model type (self-reported): microsimulation

Risk factors included (smoking status provided with details): smoking intensity

(packs/day) - heavy/moderate/light, CD4+T cell count, viral load, history of opportunistic disease and antiretroviral treatment use

Diseases: lung cancer

Outcomes: life expectancy, mortality

Sensitivity analysis: two-way

Validation: internal validation, external validation and cross-validation

Number of peer-reviewed articles in this search: 2

Related papers: 9293

Model name/first author: Parrott model

Model type (self-reported): decision tree

Risk factors included (smoking status provided with details): childhood exposure to maternal smoking, smoking status (current, former)

Diseases: COPD, CHD, stroke, lung cancer, asthma, pregnancy-related (placental

abruption, ectopic pregnancy, pre-eclampsia, placenta previa and miscarriage, infant morbidities: low infant birth weight, stillbirth, premature birth)

Outcomes: ICER, QALY, uncertainty

Sensitivity analysis: PSA

Validation: no model validation

Number of peer-reviewed articles in this search: 2

Related papers: 6768

Model name/first author: Tobacco Town

Model type (self-reported): agent-based model

Risk factors included (smoking status provided with details): smoking intensity (cigarettes/day)

Diseases: NA

Outcomes: cost, tobacco purchase behaviour

Sensitivity analysis: performed sensitivity analysis

Validation: no model validation

Number of peer-reviewed articles in this search: 2

Related papers: 7980

\section{Model name/first author: Chronic Disease Model}

Model type (self-reported): closed cohort multistate Markov model

Risk factors included (smoking status provided with details): smoking status (never, former, current)

Diseases: acute myocardial infarction, chronic heart failure, COPD, stroke (CVA), T2DM, and cancer of the lung, stomach, oesophagus, larynx, bladder, kidney, pancreas, and oral cavity

Outcomes: cost, QALY, number of quitters

Sensitivity analysis: one-way

Validation: no model validation

Number of peer-reviewed articles in this search: 1

Related papers: 117
Model name/first author: Barnett model

Model type (self-reported): a Markov model

Risk factors included (smoking status provided with details): smoking status (former, current)

Diseases: NA

Outcomes: mortality, healthcare cost, QALY, uncertainty

Sensitivity analysis: one-way, PSA

Validation: no model validation

Number of peer-reviewed articles in this search: 2

Related papers: 5960

Model name/first author: Chevreul model

Model type (self-reported): Markov state transition model

Risk factors included (smoking status provided with details): smoking status

(smoker, former smoker), diagnosed with either lung cancer, COPD or CVD such as

stroke or coronary artery disease and dead (smoker: $\geq 1$ cigarette/day)

Diseases: COPD, CVD, lung cancer

Outcomes: ICER

Sensitivity analysis: deterministic sensitivity analysis, Monte Carlo simulation

Validation: internal validation, external validation

Number of peer-reviewed articles in this search: 2

Related papers: 101102

Model name/first author: ModelHealth: Tobacco

Model type (self-reported): microsimulation

Risk factors included (smoking status provided with details): smoking status (never, former, current)

Diseases: CVD, stroke, lung cancer, respiratory disease

Outcomes: medical cost, hospitalisation, mortality and morbidity, productivity loss,

QALY, smoking prevalence

Sensitivity analysis: one-way

Validation: internal validation, external validation

Number of peer-reviewed articles in this search: 2

Related papers: 103104

Model name/first author: Population Health Impact Model

Risk factors included (smoking status provided with details): never tobacco

users, former tobacco users, current cigarette smokers, current cMRTP users, current

dual users

Diseases: COPD, IHD, stroke, lung cancer

Outcomes: mortality, cMRTP uptake

Sensitivity analysis: performed sensitivity analysis

Validation: model validated

Number of peer-reviewed articles in this search: 2

Related papers: 1852

Model name/first author: UK Health Forum simulation

Model type (self-reported): microsimulation

Risk factors included (smoking status provided with details): smoking status

(never, former, current)

Diseases: COPD, CHD, stroke, 14 cancers

Outcomes: cost, morbidity, smoking prevalence, uncertainty

Sensitivity analysis: performed sensitivity analysis

Validation: no model validation

Number of peer-reviewed articles in this search: 2

Related papers: 8182

Model name/first author: Chronic Heart Disease Policy Model

Model type (self-reported): state-transition (Markov) computer-simulation model

Risk factors included (smoking status provided with details): active smoker

or secondhand smoke exposure, systolic blood pressure, BMI, level of high-density

lipoprotein cholesterol, level of low-density lipoprotein, diabetes

Diseases: CHD and stroke

Outcomes: CHD incidence, prevalence, mortality, costs, uncertainty

Sensitivity analysis: Monte Carlo simulations

Validation: no model validation

Number of peer-reviewed articles in this search: 1

Related papers: 118 


\begin{tabular}{|c|c|}
\hline Model name/first author: Lung Cancer Policy Model & Model name/first author: Mendez model \\
\hline Model type (self-reported): state-transition microsimulation model & Model type (self-reported): Excel-based Markov model \\
\hline $\begin{array}{l}\text { Risk factors included (smoking status provided with details): smoking history } \\
\text { (length of time a person smoked and cigarettes smoked per day) }\end{array}$ & $\begin{array}{l}\text { Risk factors included (smoking status provided with details): smoking status } \\
\text { (never, former, current) }\end{array}$ \\
\hline Diseases: three cancers from any of the following five lung cancer cell types: & Diseases: NA \\
\hline adenocarcinoma (including adenocarcinoma in situ), large cell, squamous cell, small cell & Outcomes: cost, DALY, smoking prevalence \\
\hline and other & Sensitivity analysis: PSA \\
\hline Outcomes: mortality rate and cost-effectiveness & Validation: model validated \\
\hline Sensitivity analysis: performed sensitivity analysis & Number of peer-reviewed articles in this search: 1 \\
\hline Validation: model validated & Related papers: $78^{*}$ \\
\hline $\begin{array}{l}\text { Number of peer-reviewed articles in this search: } 1 \\
\text { Related papers: } 69\end{array}$ & \\
\hline
\end{tabular}

Model name/first author: Mejia model

Model type (self-reported): decision tree model used in Monte Carlo simulations

Risk factors included (smoking status provided with details): smoking status

(current cigarette user, current e-cigarette, dual user)

Diseases: NA

Outcomes: morbidity, uncertainty

Sensitivity analysis: performed sensitivity analysis

Validation: no model validation

Number of peer-reviewed articles in this search: 1

Related papers: 83

*Paper mentioned that this simulation model is based on the model developed by Mendez, Warner and Courant.

$\mathrm{BMI}$, body mass index; CHD, coronary heart disease; CMRTP, candidate modified risk tobacco products; COPD, chronic obstructive pulmonary disease; CVA, cerebrovascular accident; CVD, cardiovascular disease; DALY, disability-adjusted life year; ICER, incremental cost-effectiveness ratio; IHD, ischaemic heart disease; NA, not available; PSA, probabilistic sensitivity analysis; QALY, quality-adjusted life year; ROI, return on investment; T2DM, type 2 diabetes mellitus.

model, Chevreul model, CHD Policy Model and Jiménez model). Some models provided detailed model information. One of the SimSmoke models, in particular, provided a detailed data source and modelling diagram. ${ }^{105}$ However, none of the models provided the source code or the pseudo-code of their algorithms.

\section{Model output}

Finally, existing modelling guidelines recommend model validation, propagation of uncertainty and sensitivity analysis. ${ }^{88} 106$

First, 11 of 25 models reported result uncertainty. Furthermore, the SimSmoke model reported uncertainty in some studies but not in others. The types of uncertainty that were reflected in the reported uncertainty intervals varied widely.

Validation is used to check result accuracy. Figure 4 and online supplemental table S7 illustrate the wide gamut of validation approaches, including external validation, cross-validation and internal validation. We treated all models published in peerreviewed journals as face validated by experts during the peerreview process. Hence, we did not count face validation for plotting or reporting.

External validation, considered the strongest validation form,

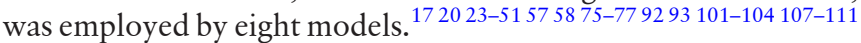
BODE $^{3}$ model and Cost-Effectiveness of Preventing AIDS Complications-US model employed cross-validations. However,

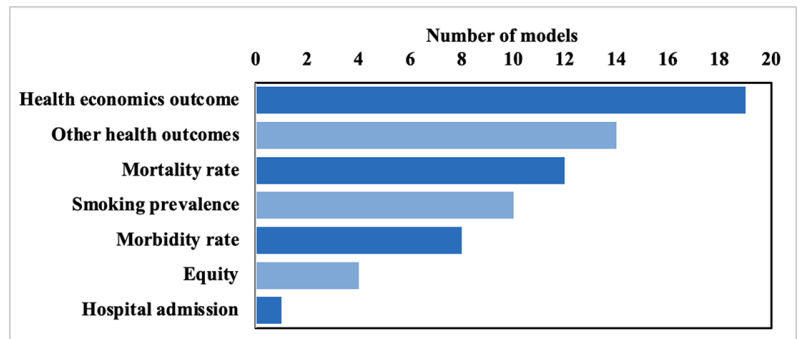

Figure 2 Occurrence of model outcome types (some models included more than one output type). some of the models did not mention the validation methods explicitly. Twelve (48\%) models did not mention any validity check (without consideration of the face validation). $.^{59-68} 79-83$ 94-100 112-118

Modellers perform a sensitivity analysis to check model outputs' variation by input uncertainty. ${ }^{106}$ All models in our review reported some sensitivity analysis using one-way, multivariable or probabilistic sensitivity analysis (PSA). Three models applied a one-way sensitivity test only. ${ }^{89-91} 103104117$ Five models applied PSA only. ${ }^{20} 253-51676875-78118$ Additionally, eight models used various approaches when testing different input parameters. 17 57-66 70-74 99-102 112-116

\section{Equity}

Given the strong socioeconomic gradient of smoking in many countries, we also considered it essential to report policy outcomes on equity. Four models reported policy equity impact. ${ }^{20} 232543-5170-74107-111117$ A range of socioeconomic status (SES) measures were used. IMPACT model used area deprivation (index of relative area-level deprivation) or education level to

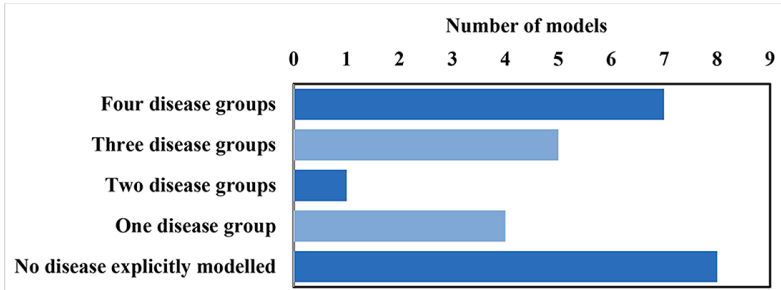

Figure 3 Occurrence of number of disease groups simulated by models. Considered disease groups: cancers, chronic obstructive pulmonary disease, cardiovascular disease and any other smokingrelated diseases. The models with no diseases explicitly modelled either calculated all-cause mortality directly from smoking status or used the number of smokers or non-smokers without explicitly modelling disease pathways. 


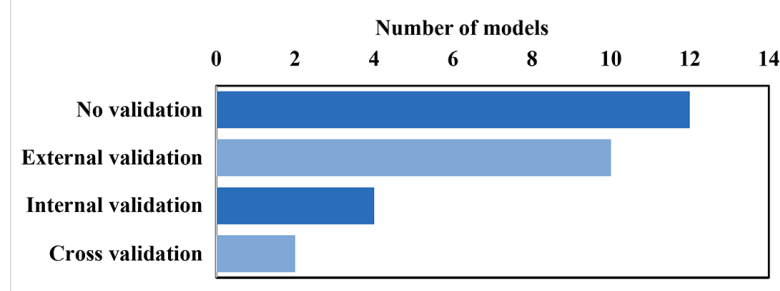

Figure 4 Occurrence of types of model validations (some models used more than one validation type).

indicate $\mathrm{SES}^{23}$ 107-111; CDM defined SES by education levels ${ }^{117}$; BODE $^{3}$ used ethnicity groups ${ }^{202543-51}$ and ECEA tobacco tax model modelled income quintiles. ${ }^{70-74}$

Developing a proof-of-concept quality assessment framework Online supplemental table S5 shows the models scored using the proposed quality assessment framework and presents the number of published articles using the model. BODE $^{3}$ was the highest scored model with one missing point on using the categorical smoking status. The models with higher quality scores were generally associated with more peer-reviewed publications (online supplemental figure S1 and online supplemental table S8).

\section{DISCUSSION}

This tobacco control PSM systematic review critically analysed existing models' strengths and weaknesses regarding data inputs, model structure and outputs. Going beyond previous systematic reviews, we then devised and proposed a tobacco control PSM quality assessment framework. This quality framework could potentially be used in future research to enable readers to better assess the quality of tobacco control PSMs.

Our systematic review confirmed the multitude of modelling techniques used in the field. It revealed a wide range of quality, with few achieving high scores. The diffusion of good modelling practices thus currently appears to be suboptimal.

All included models had been subjected to sensitivity analysis and most appropriately used population data from public or administrative registries to represent the population. ${ }^{106} 119$ However, other best practices were often lacking.

Few models adequately captured the epidemiology of smoking harms. Smoking intensity and duration are essential, ${ }^{120}$ and the risk from smoking is cumulative, especially for cancers and COPD. The risk reduction after smoking cessation is likewise gradual. In addition, considerable time lags between exposure and change in risk exist for some diseases. By ignoring these factors, many models risk overestimating the impact of the simulated tobacco control policies. ${ }^{121}$

Furthermore, around one in five models used no empirical evidence to inform policy effectiveness. Thus, risking substantial bias.

Most models provided documentation to explain technical details for readers. However, none offered the source code under an open-source licence to enable complete transparency and scrutiny. We consider this a missed opportunity for transparency and sharing good practice, avoiding unnecessary repetition of work between research groups, and enabling more rapid model development. ${ }^{14} 106122123$ Ultimately, these coding silos hinder evidenced-based health policymaking and evaluation by needlessly slowing down model development and the dispersion of good practice.
Only four models reported on the potential equity of the simulated tobacco control policies. This is despite smoking prevalence having strong socioeconomic gradients in most countries; gradients which inequitable tobacco control policies have sometimes intensified. ${ }^{121} 124$

\section{Quality assessment framework}

In developing our proof-of-concept quality assessment framework, we included nine dimensions. Each appeared feasible, being achieved in at least one tobacco control PSM. Reassuringly, the models with the highest quality scores were broadly those with a higher number of publications, although two highquality models with high publication count perhaps drove the pattern.

\section{Public health implications}

Policymakers could use this review as a registry of the currently available models. Furthermore, we propose an easy-to-use framework to assess the quality of the existing and future models, guide narratives of quality assessment during the peer-review process and foster progressively higher quality models.

Earlier guidelines powerfully informed our proposed quality assessment framework. ${ }^{123}$ However, we would suggest that most such guidelines are primarily useful for modellers rather than model users. They are lengthy (span across seven papers), challenging for non-technical users to digest and practice, and too generic to directly cover specific tobacco epidemiology characteristics (such as the cumulative nature of the risk and long lags between exposure and some diseases). These shortcomings may perhaps help explain the lack of any quality assessment in the two previous systematic reviews on tobacco models. We believe that our proposed quality assessment framework would be simple to apply directly to tobacco control PSMs and would not require the user to have any deep technical background.

The quality assessment framework we are proposing may also incentivise modelling complexity. We argue that complexity is necessary to integrate the increasingly available information, enabling richer, more accurate and comparable modelling outputs for policymakers and planners. ${ }^{124}$ Increased collaboration between modelling teams is thus urgently needed to mitigate many of the potential pitfalls of complexity and improve quality.

Organisations that facilitate collaboration among health policy modellers could play an important role. For instance, CISNET, a National Cancer Institute-funded modelling consortium, shares model common inputs and common intermediate/final outputs; modellers can then compare prediction results between different models. $^{125126}$

In the longer term, such collaboration would create a virtuous circle of modellers having a framework to support them and policymakers having consistently better models.

\section{Strengths and limitations}

Building on previous reviews, we applied broader inclusion requirements and enhanced systematic methods. Our systematic review thus offers a broader and deeper view of the current tobacco control PSM landscape.

Additionally, we went beyond the traditional methodology review to provide an easy-to-use framework for the quality assessment of existing and future models. This should facilitate the development of higher quality tobacco control PSMs and may be useful during the peer-review process. 
This review has potential limitations. First, we only analysed PSMs used in more than two studies to better represent the most actively used tobacco control PSMs. Likewise, we excluded models with more than two studies if these were all published before July 2013, as these can be found in the previous review by Feirman et al. Unavoidably, our approach has excluded some tobacco control PSMs. However, given the aims of our study, we would not expect them to have fundamentally different modelling practices than the models we included.

Second, allocating a single point in each of the nine (binary) dimensions of the quality framework was intended to be simple but risks being simplistic. However, in future real-world uses of the framework, we expect to refine these methods into more elaborate and weighted scoring schemes, perhaps tailored to the specific research aims. That further development and validation might permit an even broader and more robust assessment of model quality.

Third, due to resource constraints, we did not search for any grey literature or reports and only included studies published in English; we may thus have excluded some potentially influential models.

Finally, we included five studies funded by industry, which is liable to COI and bias. That represents a topic for further research.

\section{CONCLUSIONS}

In conclusion, we have usefully highlighted the strengths and weaknesses of tobacco control PSMs' data selection, model structure and output. We offer a nine-dimension proof-of-concept quality assessment framework to help facilitate the development of high-quality policy models in tobacco control, and perhaps more widely.

\section{What this paper adds}

\section{What is already known on this subject}

- Tobacco control policy simulation models have been used to guide tobacco control policymaking during the planning stage and the evaluation of post-implementation impact. However, despite this being a very active research area, there is no widely accepted quality assessment framework for tobacco control policy simulation models.

What this paper adds

- Analysing the methodology of published tobacco control policy simulation models potentially offers a broader and deeper view of the current policy modelling landscape.

- We offer a proof-of-concept quality assessment framework for tobacco control policy simulation models, which may guide quality assessment narratives in the peer-review process and foster higher modelling standards.

Contributors The systematic review was drafted, conducted and finalised by $\mathrm{VH}$ and $\mathrm{CK}$, with substantive contributions from AH, LH, SC, MO'F and IB. CK is the guarantor.

Funding VH is supported by the UKRI Economic and Social Research Council doctoral training award (training grant reference number: ES/P000665/1). AH is funded by a departmental studentship at the University of Liverpool and supported by the UK National Institute for Health Research (NIHR) School for Public Health (grant reference number PD-SPH-2015-10025). CK, SC, MO'F and IB are funded by UUK. IB is supported by NIHR as a Senior Investigator.

Disclaimer The views expressed are those of the author(s) and not necessarily those of the NIHR, and the Department of Health and Social Care.

Competing interests None declared.

Patient consent for publication Not required.
Ethics approval This study does not involve human participants.

Provenance and peer review Not commissioned; externally peer reviewed.

Data availability statement All data relevant to the study are included in the article or uploaded as supplemental information.

Open access This is an open access article distributed in accordance with the Creative Commons Attribution 4.0 Unported (CC BY 4.0) license, which permits others to copy, redistribute, remix, transform and build upon this work for any purpose, provided the original work is properly cited, a link to the licence is given, and indication of whether changes were made. See: https://creativecommons.org/ licenses/by/4.0/.

\section{ORCID iDs}

Vincy Huang http://orcid.org/0000-0002-2569-0701

Chris Kypridemos http://orcid.org/0000-0002-0746-9229

\section{REFERENCES}

1 McBryde ES, Meehan MT, Adegboye OA, et al. Role of modelling in COVID-19 policy development. Paediatr Respir Rev 2020;35:57-60.

2 GOV.UK. Chief medical officer annual report 2018: better health within reach. Available: https://www.gov.uk/government/publications/chief-medical-officerannual-report-2018-better-health-within-reach [Accessed 12 Jan 2020].

3 Tobacco. Available: https://www.who.int/news-room/fact-sheets/detail/tobacco [Accessed 6 Jan 2021].

4 Europe PMC. Should prioritising health interventions be informed by modelling studies? The case of cancer control in Aotearoa New Zealand - Abstract. Available: https://europepmc.org/article/med/33767491 [Accessed 2 Apr 2021].

5 Feirman SP, Donaldson E, Glasser AM, et al. Mathematical modeling in tobacco control research: initial results from a systematic review. Nicotine Tob Res 2016;18:229-42

6 Feirman S, Donaldson E, Pearson J, et al. Mathematical modelling in tobacco control research: protocol for a systematic review. BMJ Open 2015;5:e007269.

7 Singh A, Wilson N, Blakely T. Simulating future public health benefits of tobacco control interventions: a systematic review of models. Tob Control 2020. doi:10.1136/ tobaccocontrol-2019-055425. [Epub ahead of print: 25 Jun 2020].

8 Lay-Yee R, Cotterell G. The Role of Microsimulation in the Development of Public Policy. In: Janssen M, Wimmer MA, Deljoo A, eds. Policy practice and digital science. Cham: Springer International Publishing, 2015: 305-20.

9 Husereau D, Drummond M, Petrou S, et al. Consolidated Health Economic Evaluation Reporting Standards (CHEERS)--explanation and elaboration: a report of the ISPOR Health Economic Evaluation Publication Guidelines Good Reporting Practices Task Force. Value Health 2013;16:231-50.

10 Brozek JL, Canelo-Aybar C, AkI EA, et al. GRADE Guidelines 30: the GRADE approach to assessing the certainty of modeled evidence-An overview in the context of health decision-making. J Clin Epidemiol 2021;129:138-50.

11 NICE. Appendix I quality appraisal checklist - economic evaluations | methods for the development of NICE public health guidance (third edition) | guidance |. Available: https://www.nice.org.uk/process/pmg4/chapter/appendix-i-qualityappraisal-checklist-economic-evaluations [Accessed 10 Apr 2020].

12 Campbell M, McKenzie JE, Sowden A, et al. Synthesis without meta-analysis (swim) in systematic reviews: reporting guideline. BMJ 2020:368:16890.

13 Page MJ, McKenzie JE, Bossuyt PM, et al. The PRISMA 2020 statement: an updated guideline for reporting systematic reviews. BMJ 2021;372:n71.

14 Eddy DM, Hollingworth W, Caro JJ, et al. Model transparency and validation: a report of the ISPOR-SMDM modeling good research practices task Force-7. Med Decis Making 2012;32:733-43.

15 Model parameter estimation and uncertainty analysis: a report of the ISPOR-SMDM Modeling Good Research Practices Task Force Working Group-6 - PubMed. Available: https://pubmed.ncbi.nlm.nih.gov/22990087/ [Accessed 5 Aug 2020].

16 Huang V, Head A, Hyseni L, et al. Tobacco control policy simulation models: protocol for a systematic methodological review. JMIR Res Protoc 2021;10:e26854.

17 Johansson P. A model for economic evaluations of smoking cessation interventions technical report, 35

18 Weitkunat R, Lee PN, Baker G, et al. A novel approach to assess the population health impact of introducing a modified risk tobacco product. Regul Toxicol Pharmacol 2015;72:87-93.

19 Golden SD, Farrelly MC, Luke DA, et al. Comparing projected impacts of cigarette floor price and excise tax policies on socioeconomic disparities in smoking. Tob Control 2016;25:i60-6.

20 Cobiac LJ, Ikeda T, Nghiem N, et al. Modelling the implications of regular increases in tobacco taxation in the tobacco endgame. Tob Control 2015;24:e154-60.

21 Grace RC, Kivell BM, Laugesen M. Predicting decreases in smoking with a cigarette purchase task: evidence from an excise tax rise in New Zealand. Tob Control 2015;24:582-7.

22 Levy DT, Huang A-T, Currie LM, et al. The benefits from complying with the framework convention on tobacco control: a SimSmoke analysis of 15 European nations. Health Policy Plan 2014;29:1031-42. 
23 Allen K, Kypridemos C, Hyseni L, et al. The effects of maximising the UK's tobacco control score on inequalities in smoking prevalence and premature coronary heart disease mortality: a modelling study. BMC Public Health 2016;16:292.

24 Levy D, Rodríguez-Buño RL, Hu T-W, et al. The potential effects of tobacco control in China: projections from the China SimSmoke simulation model. BMJ 2014;348:g1134

25 Pearson AL, van der Deen FS, Wilson N, et al. Theoretical impacts of a range of major tobacco retail outlet reduction interventions: modelling results in a country with a smoke-free nation goal. Tob Control 2015;24:e32-8.

26 Szklo AS, Yuan Z, Levy D. Update and extension of the Brazil SimSmoke model to estimate the health impact of cigarette smoking by pregnant women in Brazil. Cad Saude Publica 2017;33:e00207416.

27 Levy DT, Fouad H, Levy J, et al. Application of the abridged SimSmoke model to four eastern Mediterranean countries. Tob Control 2016;25:413-21.

28 Levy D, Abrams DB, Levy J, et al. Complying with the framework convention for tobacco control: an application of the abridged SimSmoke model to Israel. Isr J Health Policy Res 2016;5.

29 Reynales-Shigematsu LM, Fleischer NL, Thrasher JF, et al. Effects of tobacco control policies on smoking prevalence and tobacco-attributable deaths in Mexico: the SimSmoke model. Rev Panam Salud Publica 2015;38:316-25.

30 Levy D, Mohlman MK, Zhang Y. Estimating the potential impact of tobacco control policies on adverse maternal and child health outcomes in the United States using the SimSmoke tobacco control policy simulation model. Nicotine Tob Res 2016;18:1240-9.

31 Levy DT, Meza R, Zhang Y, et al. Gauging the effect of U.S. tobacco control policies from 1965 through 2014 using SimSmoke. Am J Prev Med 2016;50:535-42.

32 Fleischer NL, Thrasher JF, Reynales-Shigematsu LM, et al. Mexico SimSmoke: how changes in tobacco control policies would impact smoking prevalence and smoking attributable deaths in Mexico. Glob Public Health 2017;12:830-45.

33 Levy DT, Wijnhoven TMA, Levy J, et al. Potential health impact of strong tobacco control policies in 11 South eastern who European region countries. Eur J Public Health 2018;28:693-701.

34 Levy DT, Mays D, Yuan Z, et al. Public health benefits from pictorial health warnings on us cigarette packs: a SimSmoke simulation. Tob Control 2017;26:649-55.

35 Maslennikova GY, Oganov RG, Boytsov SA, et al. Russia SimSmoke: the long-term effects of tobacco control policies on smoking prevalence and smoking-attributable deaths in Russia. Tob Control 2014;23:484-90.

36 Near AM, Blackman K, Currie LM, et al. Sweden SimSmoke: the effect of tobacco control policies on smoking and snus prevalence and attributable deaths. Eur J Public Health 2014;24:451-8.

37 Levy DT, Huang A-T, Currie LM, et al. The benefits from complying with the framework convention on tobacco control: a SimSmoke analysis of 15 European nations. Health Policy Plan 2014;29:1031-42.

38 Levy D, Rodriguez-Buno RL, Hu T. The Potential Effects of Tobacco Control in China: Projections from the China SimSmoke Simulation Model. In: Hu T, ed. Economics of Tobacco Control in China: From Policy Research to Practice. World Scientific Series in Global Health Economics and Public Policy. London and Singapore: World Scientific, 2016: vol. 4. 189-213. https://liverpool.idm.oclc.org/login?url=https://search. ebscohost.com/login.aspx? direct=true $\& d b=e c n \& A N=1745241 \&$ site=ehost-live \& scope=site

39 Levy DT, Yuan Z, Li Y. The US SimSmoke tobacco control policy model of smokeless tobacco and cigarette use. BMC Public Health 2018;18:696.

40 Sanna M, Gao W, Chiu Y-W, et al. Tobacco control within and beyond WHO MPOWER: outcomes from Taiwan SimSmoke. Tob Control 2020:29:36-42.

41 Levy D, Fergus C, Rudov L, et al. Tobacco policies in Louisiana: recommendations for future tobacco control investment from SimSmoke, a policy simulation model. Prevention Science 2016;17:199-207.

42 Levy DT, Lindblom EN, Fleischer NL, et al. Public health effects of restricting retail tobacco product displays and ads. Tob Regul Sci 2015;1:61-75.

43 Nghiem N, Cleghorn CL, Leung W, et al. A national Quitline service and its promotion in the mass media: modelling the health gain, health equity and cost-utility. Tob Control 2018;27:434-41.

44 Blakely T, Cobiac LJ, Cleghorn CL, et al. Health, health inequality, and cost impacts of annual increases in tobacco tax: multistate life table modeling in New Zealand. PLoS Med 2015;12:e1001856.

45 van der Deen FS, Wilson N, Cleghorn CL, et al. Impact of five tobacco endgame strategies on future smoking prevalence, population health and health system costs: two modelling studies to inform the tobacco endgame. Tob Control 2018;27:278-86.

46 Cleghorn CL, Blakely T, Kvizhinadze G, et al. Impact of increasing tobacco taxes on working-age adults: short-term health gain, health equity and cost savings. Tob Control 2018:27:e167-70

47 Singh A, Petrović-van der Deen FS, Carvalho N, et al. Impact of Tax and tobacco-free generation on health-adjusted life years in the Solomon Islands: a multistate life table simulation. Tob Control 2020;29:tobaccocontrol-2018-054861.

48 Nghiem N, Leung W, Cleghorn C, et al. Mass media promotion of a smartphone smoking cessation APP: modelled health and cost-saving impacts. BMC Public Health 2019;19:283.
49 Petrović-van der Deen FS, Wilson N, Crothers A, et al. Potential country-level health and cost impacts of legalizing domestic sale of Vaporized nicotine products. Epidemiology 2019;30:396-404.

50 Petrović-van der Deen FS, Blakely T, Kvizhinadze G, et al. Restricting tobacco sales to only pharmacies combined with cessation advice: a modelling study of the future smoking prevalence, health and cost impacts. Tob Control 2019;28:643-50.

51 Pearson AL, Cleghorn CL, van der Deen FS, et al. Tobacco retail outlet restrictions: health and cost impacts from multistate life-table modelling in a national population. Tob Control 2017;26:579-85.

52 Djurdjevic S, Lee $\mathrm{P}$, Weitkunat $\mathrm{R}$, et al. Modeling the population health impact of introducing a modified risk tobacco product into the U.S. market. Health Care 2018;6:47.

53 Hill A, Camacho OM. A system dynamics modelling approach to assess the impact of launching a new nicotine product on population health outcomes. Regul Toxicol Pharmacol 2017:86:265-78.

54 Poland B. Population modeling of modified risk tobacco products accounting for smoking reduction and gradual transitions of relative risk. Available: https:// kopernio.com/viewer?doi=10.1093\%2Fntr\%2Fntx070\&token=WzlxMDU2NzcsljEw LjEwOTMvbnRyL250eDA3MCJd.IFnWIJvqI5E8bC518Re68t4Pwxw [Accessed $18 \mathrm{Apr}$ 2020].

55 Bachand AM, Sulsky SI. A dynamic population model for estimating allcause mortality due to lifetime exposure history. Regul Toxicol Pharmacol 2013;67:246-51.

56 Muhammad-Kah RS, Pithawalla YB, Boone EL, et al. A computational model for assessing the population health impact of introducing a modified risk claim on an existing smokeless tobacco product. Int J Environ Res Public Health 2019;16:E1264.

57 Feldman I, Helgason AR, Johansson P, et al. Cost-Effectiveness of a high-intensity versus a low-intensity smoking cessation intervention in a dental setting: long-term follow-up. BMJ Open 2019;9:e030934.

58 Virtanen SE, Galanti MR, Johansson PM, et al. Economic evaluation of a brief counselling for smoking cessation in dentistry: a case study comparing two health economic models. BMJ Open 2017;7:e016375.

59 Barnett PG, Wong W, Jeffers $A$, et al. Cost-Effectiveness of extended cessation treatment for older smokers. Addiction 2014;109:314-22.

60 Barnett PG, Jeffers A, Smith MW, et al. Cost-Effectiveness of integrating tobacco cessation into post-traumatic stress disorder treatment. Nicotine Tob Res 2016;18:267-74.

61 Daly AT, Deshmukh AA, Vidrine DJ, et al. Cost-Effectiveness analysis of smoking cessation interventions using cell phones in a low-income population. Tob Control 2019;28:88-94

62 Cantor SB, Deshmukh AA, Luca NS, et al. Cost-Effectiveness analysis of smoking cessation counseling training for physicians and pharmacists. Addict Behav 2015:45:79-86.

63 Baker CL, Pietri G. A cost-effectiveness analysis of varenicline for smoking cessation using data from the eagles trial. Clinicoecon Outcomes Res 2018;10:67-74.

64 Annemans L, Marbaix S, Nackaerts K, et al. Cost-effectiveness of retreatment with varenicline after failure with or relapse after initial treatment for smoking cessation. Prev Med Rep 2015;2:189-95.

65 Kautiainen K, Ekroos H, Puhakka M, et al. Re-treatment with varenicline is a costeffective aid for smoking cessation. J Med Econ 2017:20:246-52.

66 von Wartburg M, Raymond V, Paradis PE. The long-term cost-effectiveness of varenicline (12-week standard course and $12+12$-week extended course) vs. other smoking cessation strategies in Canada. Int J Clin Pract 2014;68:639-46.

67 Jones M, Smith M, Lewis S, et al. A dynamic, modifiable model for estimating costeffectiveness of smoking cessation interventions in pregnancy: application to an RCT of self-help delivered by text message. Addiction 2019;114:353-65

68 Wu Q, Parrott S, Godfrey C, et al. Cost-Effectiveness of computer-tailored smoking cessation advice in primary care: a randomized trial (escape). Nicotine Tob Res 2014:16:270-8

69 Tramontano AC, Sheehan DF, McMahon PM, et al. Evaluating the impacts of screening and smoking cessation programmes on lung cancer in a high-burden region of the USA: a simulation modelling study. BMJ Open 2016;6:e010227.

70 Verguet S, Gauvreau CL, Mishra S, et al. The consequences of tobacco tax on household health and finances in rich and poor smokers in China: an extended costeffectiveness analysis. Lancet Glob Health 2015;3:e206-16.

71 Salti $N$, Brouwer $E$, Verguet $S$. The health, financial and distributional consequences of increases in the tobacco excise tax among smokers in Lebanon. Soc Sci Med 2016:170:161-9.

72 Global Tobacco Economics Consortium. The health, poverty, and financial consequences of a cigarette price increase among 500 million male smokers in 13 middle income countries: compartmental model study. BMJ 2018;361:k1162.

73 Verguet S, Laxminarayan R, Jamison DT. Universal public finance of tuberculosis treatment in India: an extended cost-effectiveness analysis. Health Econ 2015:24:318-32

74 Verguet S, Tarr G, Gauvreau CL, et al. Distributional benefits of tobacco Tax and smoke-free workplaces in China: a modeling study. J Glob Health 2017;7:020701.

75 Soler R, Orenstein D, Honeycutt A, et al. Community-Based interventions to decrease obesity and tobacco exposure and reduce health care costs: outcome estimates 
from communities putting prevention to work for 2010-2020. Prev Chronic Dis 2016;13:E47

76 Honeycutt A, Bradley C, Khavjou 0, et al. Simulated impacts and potential cost effectiveness of communities putting prevention to work: tobacco control interventions in 21 U.S. communities, 2010-2020. Prev Med 2019;120:100-6.

77 Homer J, Wile K, Yarnoff B, et al. Using simulation to compare established and emerging interventions to reduce cardiovascular disease risk in the United States. Prev Chronic Dis 2014;11:E195.

78 Jin L, Kenkel D, Liu F, et al. Retrospective and prospective Benefit-Cost analyses of U.S. anti-smoking policies. J Benefit Cost Anal 2015;6:154-86.

79 Combs TB, McKay VR, Ornstein J, et al. Modelling the impact of menthol sales restrictions and retailer density reduction policies: insights from tobacco town Minnesota. Tob Control 2020;29:502-9.

80 Luke DA, Hammond RA, Combs T, et al. Tobacco town: computational modeling of policy options to reduce tobacco Retailer density. Am J Public Health 2017; 107:740-6

81 Knuchel-Takano A, Hunt D, Jaccard A, et al. Modelling the implications of reducing smoking prevalence: the benefits of increasing the UK tobacco duty escalator to public health and economic outcomes. Tob Control 2018;27:e124-9.

82 Hunt D, Knuchel-Takano A, Jaccard A, et al. Modelling the implications of reducing smoking prevalence: the public health and economic benefits of achieving a 'tobacco-free' UK. Tob Control 2018;27:129-35.

83 Kalkhoran S, Glantz SA. Modeling the health effects of expanding e-cigarette sales in the United States and United Kingdom: a Monte Carlo analysis. JAMA Intern Med 2015;175:1671-80

84 Lubin JH, Couper D, Lutsey PL, et al. Risk of cardiovascular disease from cumulative cigarette use and the impact of smoking intensity. Epidemiology 2016;27:395-404.

85 Forey BA, Thornton AJ, Lee PN. Systematic review with meta-analysis of the epidemiological evidence relating smoking to COPD, chronic bronchitis and emphysema. BMC Pulm Med 2011;11:36.

86 Tammemägi MC, Church TR, Hocking WG, et al. Evaluation of the lung cancer risks at which to screen ever- and never-smokers: screening rules applied to the PLCO and NLST cohorts. PLoS Med 2014;11:e1001764.

87 Bonnie RJ, Stratton K, Kwan LY. The effects of tobacco use on health. National Academies Press (US), 2015. https://www.ncbi.nlm.nih.gov/books/NBK310413/

88 Webber L, Mytton OT, Briggs ADM, et al. The Brighton declaration: the value of non-communicable disease modelling in population health sciences. Eur J Epidemiol 2014;29:867-70.

89 Cedillo S, Sicras-Mainar A, Jiménez-Ruiz CA, et al. Budgetary impact analysis of reimbursement varenicline for the smoking-cessation treatment in patients with cardiovascular diseases, chronic obstructive pulmonary disease or type-2 diabetes mellitus: a national health system perspective. Eur Addict Res 2017;23:7-18.

90 Jiménez-Ruiz CA, Solano-Reina S, Signes-Costa J, et al. Budgetary impact analysis on funding smoking-cessation drugs in patients with COPD in Spain. Int J Chron Obstruct Pulmon Dis 2015;10:2027-36.

91 Rejas-Gutiérrez J, López-Ibáñez de Aldecoa A, Casasola M, et al. Economic evaluation of combining Pharmaco- and behavioral therapies for smoking cessation in an occupational medicine setting. J Occup Environ Med 2019;61:318-27.

92 Reddy KP, Parker RA, Losina E, et al. Impact of cigarette smoking and smoking cessation on life expectancy among people with HIV: a US-Based modeling study. J Infect Dis 2016:214:1672-81.

93 Reddy KP, Kong CY, Hyle EP, et al. Lung cancer mortality associated with smoking and smoking cessation among people living with HIV in the United States. JAMA Intern Med 2017:177:1613-21.

94 Holm AL, Brønnum-Hansen H, Robinson KM, et al. Assessment of health impacts of decreased smoking prevalence in Copenhagen: application of the DYNAMO-HIA model. Scand J Public Health 2014;42:409-16.

95 Leão T, Perelman J, Clancy L, et al. Economic evaluation of five tobacco control policies across seven European countries. Nicotine Tob Res 2020;22:1202-9.

96 Fischer F, Kraemer A. Health Impact Assessment for Second-Hand Smoke Exposure in Germany--Quantifying Estimates for Ischaemic Heart Diseases, COPD, and Stroke. Int J Environ Res Public Health 2016;13:198.

97 Füssenich K, Nusselder WJ, Lhachimi SK, et al. Potential gains in health expectancy by improving lifestyle: an application for European regions. Popul Health Metr 2019:17:N.PAG-N.PAG.

98 Lhachimi SK, Nusselder WJ, Smit HA, et al. Potential health gains and health losses in eleven EU countries attainable through feasible prevalences of the life-style related risk factors alcohol, BMI, and smoking: a quantitative health impact assessment. BMC Public Health 2016;16:734.

99 Baker CL, Ding Y, Ferrufino CP, et al. A cost-benefit analysis of smoking cessation prescription coverage from a US payer perspective. Clinicoecon Outcomes Res 2018;10:359-70.

100 Baker CL, Ferrufino CP, Bruno M, et al. Estimated budget impact of adopting the Affordable care act's required smoking cessation coverage on United States healthcare payers. Adv Ther 2017;34:156-70.
101 Cadier B, Durand-Zaleski I, Thomas D, et al. Cost effectiveness of free access to smoking cessation treatment in France considering the economic burden of smoking-related diseases. PLoS One 2016;11:e0148750.

102 Chevreul K, Cadier B, Durand-Zaleski I, et al. Cost effectiveness of full coverage of the medical management of smoking cessation in France. Tob Control 2014:23:223-30

103 Maciosek MV, LaFrance AB, Dehmer SP, et al. Health benefits and cost-effectiveness of brief clinician tobacco counseling for youth and adults. Ann Fam Med 2017; 15:37-47.

104 Maciosek MV, LaFrance AB, St Claire A, et al. Twenty-Year health and economic impact of reducing cigarette use: Minnesota 1998-2017. Tob Control 2020;29:564-9.

105 Levy D, Fergus C, Rudov L, et al. Tobacco policies in Louisiana: recommendations for future tobacco control investment from SimSmoke, a policy simulation model. Prev Sci 2016:17:199-207.

106 Weinstein MC, O'Brien B, Hornberger J, et al. Principles of good practice for decision analytic modeling in health-care evaluation: report of the ISPOR Task Force on Good Research Practices--Modeling Studies. Value Health 2003;6:9-17.

107 Björck L, Rosengren A, Winkvist A, et al. Changes in dietary fat intake and projections for coronary heart disease mortality in Sweden: a simulation study. PLoS One 2016;11:e0160474.

108 Sözmen K, Unal B, Capewell S, et al. Estimating diabetes prevalence in turkey in 2025 with and without possible interventions to reduce obesity and smoking prevalence, using a modelling approach. Int J Public Health 2015;60 Suppl 1:13-21.

109 O'Flaherty M, Bandosz P, Critchley J, et al. Exploring potential mortality reductions in 9 European countries by improving diet and lifestyle: a modelling approach. Int J Cardiol 2016;207:286-91.

110 Sahan C, Sozmen K, Unal B, et al. Potential benefits of healthy food and lifestyle policies for reducing coronary heart disease mortality in Turkish adults by 2025: a modelling study. BMJ Open 2016;6:e011217.

111 Scholes S, Bajekal M, Norman P, et al. Quantifying policy options for reducing future coronary heart disease mortality in England: a modelling study. PLoS One 2013:8:e69935.

112 Trapero-Bertran M, Muñoz C, Coyle K, et al. Cost-Effectiveness of alternative smoking cessation scenarios in Spain: results from the EQUIPTMOD. Addiction 2018;113 Suppl 1:65-75.

113 Huber MB, Präger M, Coyle K, et al. Cost-Effectiveness of increasing the reach of smoking cessation interventions in Germany: results from the EQUIPTMOD. Addiction 2018;113 Suppl 1:52-64.

114 Németh B, Józwiak-Hagymásy J, Kovács G, et al. Cost-Effectiveness of possible future smoking cessation strategies in Hungary: results from the EQUIPTMOD. Addiction 2018;113 Suppl 1:76-86.

115 Coyle K, Coyle D, Lester-George A, et al. Development and application of an economic model (EQUIPTMOD) to assess the impact of smoking cessation. Addiction 2018;113 Suppl 1:7-18

116 Cheung K-L, Wijnen BFM, Hiligsmann M, et al. Is it cost-effective to provide Internetbased interventions to complement the current provision of smoking cessation services in the Netherlands? An analysis based on the EQUIPTMOD. Addiction 2018;113 Suppl 1:87-95.

117 Over EAB, Feenstra TL, Hoogenveen RT, et al. Tobacco control policies specified according to socioeconomic status: health disparities and cost-effectiveness. Nicotine Tob Res 2014;16:725-32.

118 Konfino J, Ferrante D, Mejia R, et al. Impact on cardiovascular disease events of the implementation of Argentina's national tobacco control law. Tob Control 2014;23:e6.

119 Nuijten MJ. The selection of data sources for use in modelling studies. Pharmacoeconomics 1998;13:305-16.

120 Leffondré K, Abrahamowicz M, Siemiatycki J, et al. Modeling smoking history: a comparison of different approaches. Am J Epidemiol 2002:156:813-23.

121 Hoogenveen RT, van Baal PH, Boshuizen HC, et al. Dynamic effects of smoking cessation on disease incidence, mortality and quality of life: the role of time since cessation. Cost Eff Resour Alloc 2008;6:1

122 Roberts M, Russell LB, Paltiel AD, et al. Conceptualizing a model: a report of the ISPOR-SMDM Modeling Good Research Practices Task Force--2. Value Health 2012;15:804-11.

123 Caro JJ, Briggs AH, Siebert U, et al. Modeling good research practices--overview: a report of the ISPOR-SMDM Modeling Good Research Practices Task Force-1. Med Decis Making 2012;32:667-77.

124 White M, Adams J, Heywood P. How and why do interventions that increase health overall widen inequalities within populations? health, inequality and society. Bristol: Policy Press, 2009: 12

125 CISNET: about. Available: https://cisnet.cancer.gov/about/index.html [Accessed 27 Apr 2021].

126 Executive summary | JNCI Monographs | Oxford academic. Available: https:// academic-oup-com.liverpool.idm.oclc.org/jncimono/article/2006/36/1/917189 [Accessed 15 May 2021]. 